\title{
Radiotherapy and Tyrosine Kinase Inhibitors in Stage IV Non-small Cell Lung Cancer: Real-life Experience
}

\author{
PAOLO BORGHETTI ${ }^{1}$, MARCO LORENZO BONU $^{2}$, ELISA ROCA $^{3}$, SARA PEDRETTI $^{1}$, \\ EMILIANO SALAH ${ }^{2}$, ANNA BAIGUINI $^{2}$, DIANA GRECO ${ }^{2}$, LUCA TRIGGIANI $^{2}$, MARTA MADDALO $^{1}$, \\ NICCOLÒ GIAJ LEVRA ${ }^{4}$, FILIPPO ALONGI ${ }^{4}$, STEFANO MARIA MAGRINI ${ }^{1}$ and MICHELA BUGLIONE ${ }^{1}$ \\ ${ }^{1}$ Department of Radiation Oncology, Olindo Alberti Radiotherapy Institute, Brescia, Italy; \\ ${ }^{2}$ Department of Radiation Oncology, Brescia University, Brescia, Italy; \\ ${ }^{3}$ Department of Medical Oncology, Spedali Civili Hospital, Brescia, Italy; \\ ${ }^{4}$ Department of Radiation Oncology, Sacro Cuore Hospital, Negrar, Italy
}

\begin{abstract}
Aim: To investigate the role of conventional radiotherapy $(R T)$ and stereotactic body radiotherapy (SBRT) in patients with epidermal growth factor (EGFR)-mutant or anaplastic lymphoma kinase (ALK) rearrangement-positive metastatic non-small cell lung cancer (NSCLC). Patients and Methods: Fifty patients with EGFR-mutated or ALK rearrangement-positive NSCLC were treated at our Institution. Radiotherapy was delivered before, after or concomitantly with tyrosine kinase inhibitors (TKIs). Acute toxicities and overall survival $(O S)$ were assessed. Results: Radiotherapy was performed within 30 days before TKI, concomitantly with TKI and within 30 days after TKI in eight (16\%), 33 (66\%) and $9(18 \%)$ cases, respectively. The median duration of TKI therapy in the whole series was 11.9 months. The median OS was 19.3 months and 1 - and 2-year OS was $71.5 \%$ and $36.5 \%$, respectively. The group treated with SBRT had a significant benefit in terms of OS $(p=0.043)$. Only two grade 3 toxicities were reported. Conclusion: RT concomitantly or close to TKI administration in stage IV NSCLC was shown to be feasible and safe. Intriguing data on $O S$ were also reported.
\end{abstract}

The treatment-of-choice for patients with stage IV epidermal growth factor $(E G F R)$-mutant or anaplastic lymphoma kinase $(A L K)$-rearranged NSCLC consists of oral tyrosine kinase inhibitors, such as anti-EGFR (gefitinib, erlotinib, afatinib, osimertinib) or anti-ALK (crizotinib, ceritinib, alectinib) tyrosine kinase inhibitors(TKIs). (TKIs), which have

This article is freely accessible online.

Correspondence to: Paolo Borghetti, Department of Radiation Oncology, Spedali Civili of Brescia, P.le Spedali Civili 1, 25124, Brescia, Italy. Tel: +39 0303995271, e-mail: paolobor82@yahoo.it

Key Words: NSCLC, TKI, stereotactic radiotherapy. replaced cytotoxic chemotherapy as first-line treatment (1, 2). In these patients, the adoption of TKIs allows for median progression-free (PFS) and overall (OS) survival ranging between 8-13 months and 18-25 months, respectively $(3,4)$.

Almost all patients eventually develop progressive disease, requiring for further treatment. The standard strategy is to switch to a second-line chemotherapy or, when indicated, to a new-generation TKI. Alternatively, in cases of oligoprogression, especially for asymptomatic cases and brain progression, it is now more and more accepted to continue with the first-line therapy and to treat the new sites of progression with a local therapy, such as radiotherapy (RT) (5-9). Oligoprogression represents a condition in which a large part of the disease burden is controlled by systemic therapy, except for a few small sites of involvement, which probably acquired resistance to the drug (10).

There is also a biological rationale underlying the association of local RT (possibly with ablative doses) with TKI inhibitors; some pre-clinical studies showed that TKI down-regulated the proliferative signals triggered by RT (such as radiation-induced autophosphorylation of EGFR. This suggests a potential radiosensitizing effect of TKIs (11).

Unfortunately, despite the great interest in recent years, there is still a lack of data on the benefits and potential sideeffects of the association of the two treatments. We, therefore, conducted a retrospective analysis of patients treated at our Institution with conventional-RT or stereotactic body radiotherapy (SBRT) combined with TKIs for EGFRmutant or $A L K$ rearrangement-positive stage IV NSCLC.

\section{Patients and Methods}

Using an Institutional query system, we identified all patients treated with RT from January 2010 to December 2016 at our Institution concomitantly with TKIs for EGFR-mutant or ALK rearrangement-positive stage IV NSCLC. Inclusion criteria of the current retrospective analysis were: (i) patients with stage 
IV NSCLC receiving anti-EGFR or anti-ALK TKIs; (ii) oligoprogressive disease, defined as $\leq 4$ new metastatic lesions at the time of presentation for radiotherapy; (iii) multi-progressive disease, defined as $>4$ new lesions, according to Institutional policy, despite some consideration of oligoprogressive disease as up to six new lesions (10).

EGFR and ALK status. Mutation analysis was conducted by extracting DNA from tissue biopsy and identifying EGFR exon 19 deletion and exon 21 L858R mutations by standard sequencing and fragment analysis, while fluorescence in situ testing was used to detect $A L K$ gene translocations.

TKI administration. When RT was delivered concomitantly with TKIs, the dose prescriptions were as follows: Gefitinib: $250 \mathrm{mg} /$ day, erlotinib: $150 \mathrm{mg} /$ day, crizotinib: $500 \mathrm{mg} /$ day, and osimertinib: 80 $\mathrm{mg} / \mathrm{day}$, and continued after RT until disease progression or unacceptable toxicity (considered grade 3 ).

Radiation treatment. We included patients treated with RT during TKI therapy or up to 30 days before or after TKI administration. According to these criteria, patients were divided into three groups: Group A: those who underwent RT no more than 30 days before the beginning of the drug; group B: those who underwent RT no more than 30 days after the definitive suspension of TKI therapy; and group C: those who underwent RT during the administration of TKI. Doses and fractionation of RT depended on the type of disease progression (oligoprogression $v s$. multi-progressive disease), sites of progression (brain $v s$. visceral) and clinical presentation (symptomatic $v s$. asymptomatic).

Outcomes and statistical analysis. To summarize the most relevant features of the clinical variables, descriptive statistics were calculated. The primary endpoint was therapy tolerability, the secondary endpoint was the OS, defined as the time from the date of the beginning of drug treatment to the date of death (any cause) or until the date of the last follow-up. All toxicities reported in the medical records were scored using the Common Terminology Criteria for Adverse Events rating scale (CTCAE 4.0) (11). Toxicity related to RT was defined as adverse events occurring within 90 days at the site of irradiation. Clinical and therapeutic characteristics were analyzed with Chi square test $(p<0.05)$. OS was estimated with Kaplan-Meier curves and potential factors affecting OS were investigated at univariate analysis with the log-rank test $(p<0.05)$. The statistical analysis was performed with SPSS (version 22.0; IBM Corp., Armonk, NY, USA).

\section{Results}

Between January 12010 and December 31 2016, 102 patients with a diagnosis of $E G F R$-mutant or $A L K$-rearranged NSCLC who underwent RT were identified. Fifty-two patients were excluded from the study because they had RT more than 30 days from the start or the end of TKI prescription. The total number of patients available for analysis was therefore 50 . Their median age was 65 (range=30-84) years and Eastern Cooperative Oncology Group Performance Status was 0 or 1 in $16(32 \%)$ and $29(58 \%)$ cases, respectively. At the beginning of RT, the pattern of progression was defined as
Table I. Description of the series.

\begin{tabular}{lc}
\hline Characteristic & $\mathrm{N}(\%)$ \\
\hline Age (median 65 years) & \\
$\leq 65$ Years & $26(52)$ \\
$>65$ Years & $24(48)$ \\
Performance status & $16(32)$ \\
0 & $29(58)$ \\
1 & $5(10)$ \\
2 & \\
No. of sites treated with RT & $11(22)$ \\
$\leq 4$ & $39(78)$ \\
$>4$ & $27(54)$ \\
Previous CHT & $15(30)$ \\
0 & $8(16)$ \\
1 & \\
2 & $9(18)$ \\
RT schedule & $41(82)$ \\
SBRT & \\
No SBRT & $27(54)$ \\
RT target & $19(38)$ \\
Brain & $4(8)$ \\
Bone & \\
Other & $28(56)$ \\
RT aim & $13(26)$ \\
Symptomatic & $9(18)$ \\
Palliative & \\
Ablative &
\end{tabular}

RT: Radiation therapy, CHT: chemotherapy, SBRT: stereotactic body radiotherapy.

oligoprogression in 11 patients $(22 \%)$, and multi-progression in 39 patients $(78 \%)$. The majority of the patients $(54 \%)$ were naïve to previous chemotherapy, $15(30 \%)$ were treated with a first chemotherapy line, eight patients (16\%) had received two chemotherapy lines before TKI. Patients characteristics are summarized in Table I.

Thirty-four patients were treated with gefitinib, nine with crizotinib, four with erlotinib and three with other TKIs. SBRT was given in nine cases (18\%) and mild hypofractionated RT in the remaining 41 patients (82\%). RTtreated disease sites were the brain for 27 patients $(57 \%)$, bone in 19 cases (38\%) and other sites (only four cases). RT had ablative aim only for nine cases $(18 \%)$.

In regard to the timing of RT, eight patients $(16 \%)$ were classified into group A, nine (18\%) in group B and $33(66 \%)$ in group $\mathrm{C}$. The median duration of TKI administration was 11.9 months (range=0.4-59.1 months). Analyzing the specific groups, the median duration of TKI administration was 9.7, $8.3 \mathrm{~s}$ and 14.2 months, for groups A-C, respectively. Additionally, the median duration of administration of the drug after RT in group C was 4.4 months (range=0.3-49.4 months). Figure 1 summarizes the timing of TKI-RT in the series and in the three different groups. 


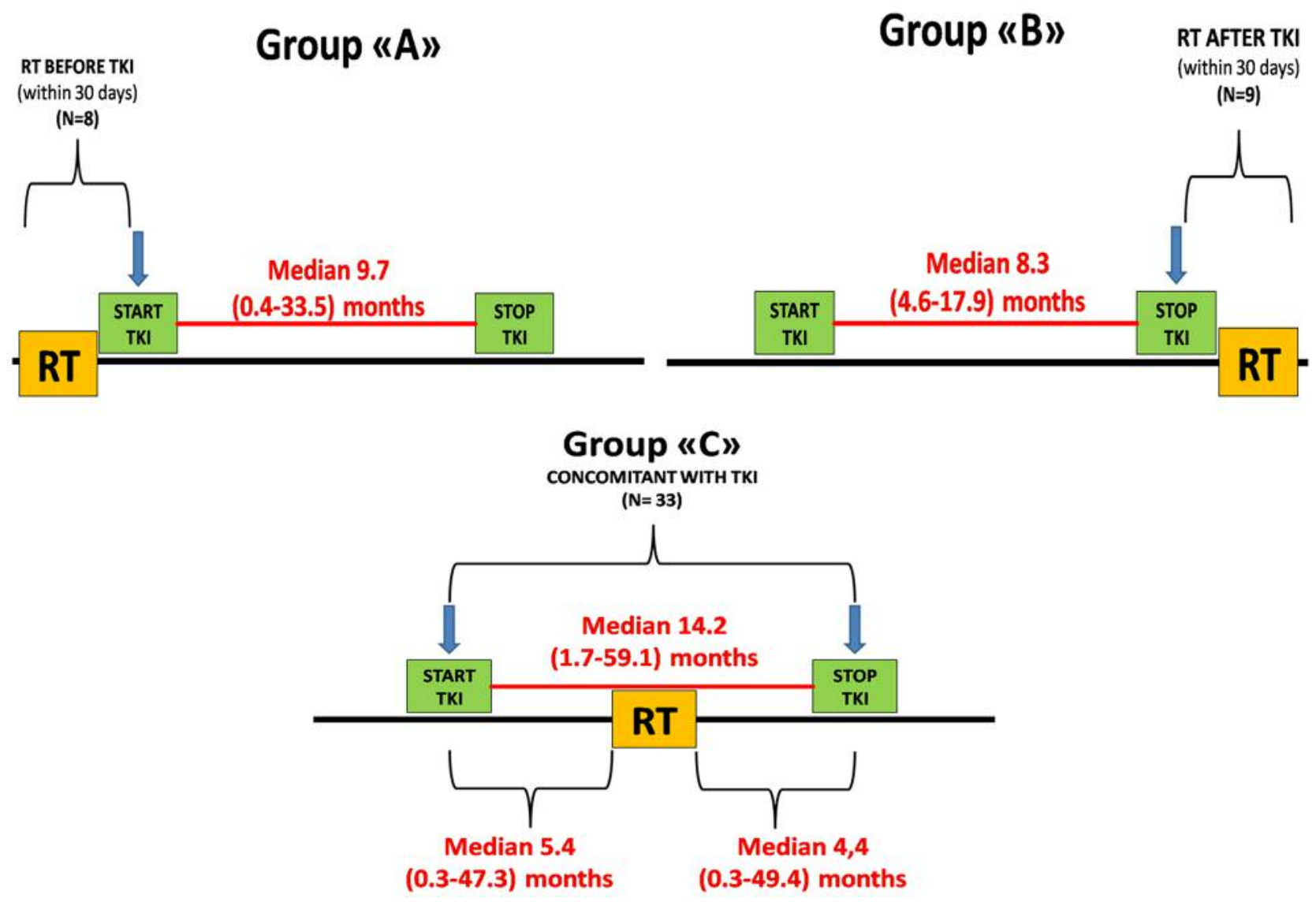

Figure 1. Timing of radiotherapy (RT) and tyrosine kinase inhibitors (TKIs) in the three treatment groups: Group A: Those who underwent RT no more than 30 days before the beginning of TKI; group B: those who underwent RT no more than 30 days after the definitive suspension of TKI therapy; and group $C$ : those who underwent $R T$ during the administration of TKI.

The median follow-up was 16 (range=1-58.9) months and was calculated from the beginning of systemic therapy to the last follow-up visit or death from any cause. The median OS was 19.3 months and 1 - and 2-year OS was $71.5 \%$ and $36.5 \%$, respectively. At univariate analysis, SBRT was associated with better OS $(p=0.043)$. No other clinical or therapeutic variable significantly affected OS.

Tolerability outcomes. In twenty-nine patients (38\%), no adverse event was recorded. In the remaining cases (31, $62 \%$ ), the following toxicity was registered: 17 patients experienced exacerbation of pain during RT (grade 1-2); 14 patients experienced transitory exacerbation of neurological signs/symptoms of brain metastasis such as headache, drowsiness, confusion, nausea and emesis during RT treatment; only two events were grade 3 . The treatment was never suspended because of an adverse event. No skin rash was observed. Adverse events are summarized in Table II.
Table II. Acute toxicity (no. of events)

\begin{tabular}{lrcccc}
\hline Toxicity & Grade 1 & Grade 2 & Grade 3 & Grade 4 & Grade 5 \\
\hline Neurological symptoms & 5 & 7 & 2 & 0 & 0 \\
Pain & 10 & 7 & 0 & 0 & 0 \\
Other & 0 & 0 & 0 & 0 & 0 \\
\hline
\end{tabular}

\section{Discussion}

There is emerging biological evidence, as described in preclinical studies, of the possible synergistic effect of TKIs given concurrently with RT at several levels, including cellcycle kinetics, apoptosis induction, and the targeting of accelerated cellular repopulation. The potential relationship between EGFR signaling and DNA damage repair is also supported by new data regarding the inhibition of RAD51 
recombinase expression. In detail, anti-EGFR and RT induce accumulation of tumor cells in $G_{1}$ and $G_{2}-M$ phases, respectively, with a reduction of cells in S-phase. When combined with RT, TKIs promotes a further reduction in the $S$-phase fraction, enhances the induction of apoptosis, inhibits EGFR autophosphorylation and expression of RAD51 following radiation exposure, thus promoting an increase in radiosensitivity (12-14).

The combination treatment with radiotherapy and TKIs does not seem to be associated with a worse toxicity in relation to RT alone or TKI alone, as suggested by other authors (15). Currently the indications for this strategy are increasing, in particular, for patients with oligometastatic disease with visceral and brain metastasis, diffuse brain metastasis and symptomatic systemic progression. Moreover, in all cases of systemic asymptomatic progression, local treatments might be considered in order to limit the malignant potential of TKI-resistant sites as sources of further disease dissemination. This is coherent with the available evidence, as the updated 2017 National Comprehensive Cancer Network (NCCN) guidelines show (16).

Some studies have demonstrated the benefit of the association of local and systemic treatments in this clinical setting, the phase II trial conducted by Iyengar et al. (5) combined SBRT with erlotinib in patients with NSCLC and metastatic lesions investigating PFS at 6 months as the principal endpoint. PFS and OS were superior in the erlotinib/RT group compared to systemic therapy alone. In more than half of the patients, no $E G F R$ mutations were found, leading the authors to conclude that the prolonged PFS was attributable to SBRT. Gan et al. proposed SBRT for all oligometastatic foci in patients with $A L K$-rearranged NSCLC in progression during crizotinib, finding no grade 3-5 toxicity, a mean crizotinib therapy duration of 28 months and a 1- and 2-year OS rates of 86 and 57\%, respectively (6). Despite these encouraging results, it is still not clear if SBRT for oligoprogressive NSCLC disease could change the natural history of the disease or whether the oligometastatic state represents a manifestation of a less aggressive biological behavior itself. The ongoing SABR-Comet trial will try to answer this question randomizing patients with oligoprogressive disease to standard of care versus SRT to all oligometastatic foci (17).

A special consideration must be made for brain metastasis. About $20-40 \%$ of patients with NSCLC develop brain metastases during the history of their illness (18). Historically, whole-brain radiation therapy (WBRT) alone or in combination with surgery and stereotactic radiotherapy has been the standard of care for brain metastases. Recent data examining survival in patients with brain metastasis in a population selected for EGFR mutations showed survival rates of 14 to 17 months from the time of brain metastasis development. Yet the treatment of brain metastasis in patients with a driver mutation remains controversial. Despite the evidence of a lower rate of central nervous system (CNS) progression in patients treated with EGFR-TKI than in those receiving chemotherapy, brain progression is still the most common site of failure in patients with $A L K$-rearranged NSCLC during TKI therapy and remains the most important event with an impact on prognosis (19). Recent phase II trials, published in 2012 and 2013, demonstrated the activity of EGFR-TKIs against brain metastasis in a very selected group of patients with EGFR-mutant NSCLC without the upfront use of RT $(20,21)$. Nevertheless, it is important to underline that the cerebrospinal fluid (CSF) penetration ratio (defined as concentration in CSF/concentration in blood) of erlotinib ranges between $2.5 \%$ to $13 \%$ and for gefitinib from $<1 \%$ to $10 \%$. Thus, despite preclinical data showing encouraging results in terms of CNS efficacy of second- and third-generation TKIs, CNS penetration of first-generation inhibitors seems to remain suboptimal (22).

An important emerging issue regards the possibility of postponing RT at the time of diagnosis of brain metastasis in patients naïve to TKIs. A series compared upfront erlotinib versus upfront WBRT or SBRT (both eventually followed by erlotinib) in 110 patients affected by EGFR-mutated NSCLC naïve to TKI with newly diagnosed brain metastasis. OS did not differ significantly between the erlotinib-treated and WBRT-treated groups, with a median OS of 26 months and 35 months, respectively ( $p=0.62)$ The SBRT -treated group had significantly longer OS than the erlotinib-treated group, with a median of 64 months $(p=0.006)$. Better local control was found in both groups treated with upfront RT. In the WBRT group, the administration of erlotinib within 2 months of WBRT was associated with improved intracranial control on univariate analysis (23). A recent publication compared treatment outcomes between TKI monotherapy versus RT plus TKIs in a cohort of 133 patients with a diagnosis of stage IV NSCLC with brain metastasis, finding a better median intracranial PFS (16.0 vs. 11.5 months, $p=0.017)$ and a better OS (22vs. 15 months $p=0.015)$ in the RT plus TKI group. Interestingly, patients harboring EGFR exon 21 mutations seemed to benefit more from the association (24).

Finally, Magnusson et al. in a large multicenter retrospective series of 351 patients compared three different approaches for first diagnosis of brain metastasis in EGFRmutant NSCLC naïve to TKIs: SBRT followed by TKI, WBRT followed by TKI and TKI alone with deferral of RT at progression. The median OS for the SBRT $(n=100)$, WBRT $(n=120)$, and EGFR-TKI $(n=131)$ cohorts was 46, 30, and 25 months, respectively $(p<0.001)$, leading to speculation that in oligoprogressive and multi-progressive settings, the major benefits are derived from the treatment associations, and that deferral of RT for brain metastasis could be associated with inferior OS (25). 
Our series shows that RT combined with TKIs is a welltolerated and promising treatment option in terms of survival, particularly when stereotactic RT with ablative aim is applied and when RT is given concomitantly with TKI. Oligoprogressive disease represents a relatively small fraction of the series and RT was proposed not only with an ablative intent, but also with a palliative-symptomatic one. For those reasons, our series represents a more "real-world" picture of the treatment of patients with stage IV NSCLC with a driver mutation. In addition, our data suggest that performing RT concomitantly and without suspension of TKI may extend the duration of drug administration, potentially leading to delaying the switch to a second-line systemic therapy. Despite most treatments being performed in a context of multiprogressive disease with palliative-symptomatic aim, OS was similar to that reported in the TKI registration studies, that notoriously consider a strictly selected group of patients (3, 4). This led us to speculate that this combination treatment could provide an advantage in terms of survival, without increasing acute toxicity, not only in those with a low burden of disease but also in a non-oligoprogressive disease setting.

\section{Conclusion}

Our study contributes to enrich the rapidly increasing literature about a very challenging setting. Local therapy such radiotherapy can contribute to optimizing the management of NSCLC with a driver mutation, in an ablative, but also palliative-symptomatic setting. Our series, in line with the current pre-clinical and clinical evidence, suggests that at progression, a strategy combining RT and systemic TKI therapy could provide major benefit and therefore must always be considered.

\section{References}

1 Burotto M, Manasanch EE, Wilkerson J and Fojo T: Gefitinib and erlotinib in metastatic non-small cell lung cancer: a metaanalysis of toxicity and efficacy of randomized clinical trials. Oncologist 20: 400-410, 2015.

2 Masters GA, Temin S, Azzoli CG, Giaccone G, Baker S Jr., Brahmer JR, Ellis PM, Gajra A, Rackear N, Schiller JH, Smith TJ, Strawn JR, Trent D, Johnson DH and American Society of Clinical Oncology Clinical P: Systemic Therapy for Stage IV Non-SmallCell Lung Cancer: American Society of Clinical Oncology Clinical Practice Guideline Update. J Clin Oncol 33: 3488-3515, 2015.

3 Lee CK, Brown C, Gralla RJ, Hirsh V, Thongprasert S, Tsai CM, Tan EH, Ho JC, Chu da T, Zaatar A, Osorio Sanchez JA, Vu VV, Au JS, Inoue A, Lee SM, Gebski V and Yang JC: Impact of EGFR inhibitor in non-small cell lung cancer on progressionfree and overall survival: a meta-analysis. J Natl Cancer Inst 105: 595-605, 2013.

4 Rosell R, Carcereny E, Gervais R, Vergnenegre A, Massuti B, Felip E, Palmero R, Garcia-Gomez R, Pallares C, Sanchez JM, Porta R, Cobo M, Garrido P, Longo F, Moran T, Insa A, De Marinis F, Corre R, Bover I, Illiano A, Dansin E, de Castro J,
Milella M, Reguart N, Altavilla G, Jimenez U, Provencio M, Moreno MA, Terrasa J, Munoz-Langa J, Valdivia J, Isla D, Domine M, Molinier O, Mazieres J, Baize N, Garcia-Campelo R, Robinet G, Rodriguez-Abreu D, Lopez-Vivanco G, Gebbia V, Ferrera-Delgado L, Bombaron P, Bernabe R, Bearz A, Artal A, Cortesi E, Rolfo C, Sanchez-Ronco M, Drozdowskyj A, Queralt C, de Aguirre I, Ramirez JL, Sanchez JJ, Molina MA, Taron M, Paz-Ares L, Spanish Lung Cancer Group in collaboration with Groupe Francais de P-C and Associazione Italiana Oncologia T: Erlotinib versus standard chemotherapy as first-line treatment for European patients with advanced EGFR mutation-positive non-small-cell lung cancer (EURTAC): a multicentre, openlabel, randomised phase 3 trial. Lancet Oncol 13: 239-246, 2012.

5 Iyengar P, Kavanagh BD, Wardak Z, Smith I, Ahn C, Gerber DE, Dowell J, Hughes R, Abdulrahman R, Camidge DR, Gaspar LE, Doebele RC, Bunn PA, Choy H and Timmerman R: Phase II trial of stereotactic body radiation therapy combined with erlotinib for patients with limited but progressive metastatic non-small-cell lung cancer. J Clin Oncol 32: 3824-3830, 2014.

6 Gan GN, Weickhardt AJ, Scheier B, Doebele RC, Gaspar LE, Kavanagh BD and Camidge DR: Stereotactic radiation therapy can safely and durably control sites of extra-central nervous system oligoprogressive disease in anaplastic lymphoma kinasepositive lung cancer patients receiving crizotinib. Int J Radiat Oncol Biol Phys 88: 892-898, 2014.

7 Yu HA, Sima CS, Huang J, Solomon SB, Rimner A, Paik P, Pietanza MC, Azzoli CG, Rizvi NA, Krug LM, Miller VA, Kris MG and Riely GJ: Local therapy with continued EGFR tyrosine kinase inhibitor therapy as a treatment strategy in EGFR-mutant advanced lung cancers that have developed acquired resistance to EGFR tyrosine kinase inhibitors. J Thorac Oncol 8: 346-351, 2013.

8 Weickhardt AJ, Scheier B, Burke JM, Gan G, Lu X, Bunn PA Jr., Aisner DL, Gaspar LE, Kavanagh BD, Doebele RC and Camidge DR: Local ablative therapy of oligoprogressive disease prolongs disease control by tyrosine kinase inhibitors in oncogene-addicted non-small-cell lung cancer. J Thorac Oncol 7: 1807-1814, 2012.

9 Besse B, Adjei A, Baas P, Meldgaard P, Nicolson M, Paz-Ares L, Reck M, Smit EF, Syrigos K, Stahel R, Felip E, Peters S, Panel M and Esmo: 2nd ESMO Consensus Conference on Lung Cancer: non-small-cell lung cancer first-line/second and further lines of treatment in advanced disease. Ann Oncol 25: 14751484, 2014.

10 Hellman S and Weichselbaum RR: Oligometastases. J Clin Oncol 13: 8-10, 1995.

11 National Cancer Institute NIoH, U.S. Department of Health and Human Services: Common Terminology Criteria for Adverse Events (CTCAE) Version 4.0. NIH publication \# 09-7473 2009.

12 Schmidt-Ullrich RK, Valerie K, Fogleman PB and Walters J: Radiation-induced autophosphorylation of epidermal growth factor receptor in human malignant mammary and squamous epithelial cells. Radiat Res 145: 81-85, 1996.

13 Zhuang H, Wang J, Zhao L, Yuan Z and Wang P: The theoretical foundation and research progress for WBRT combined with erlotinib for the treatment of multiple brain metastases in patients with lung adenocarcinoma. Int J Cancer 133: 22772283,2013 . 
14 Chinnaiyan P, Huang S, Vallabhaneni G, Armstrong E, Varambally S, Tomlins SA, Chinnaiyan AM and Harari PM: Mechanisms of enhanced radiation response following epidermal growth factor receptor signaling inhibition by erlotinib (Tarceva). Cancer Res 65: 3328-3335, 2005.

15 Jiang T, Min W, Li Y, Yue Z, Wu C and Zhou C: Radiotherapy plus EGFR TKIs in non-small cell lung cancer patients with brain metastases: an update meta-analysis. Cancer Med 5: 1055$1065,2016$.

16 National Comprehensive Cancer Netwoork (NCCN): NSCLC guidelines. NCCN pubblication 2017.

17 Palma DA, Haasbeek CJ, Rodrigues GB, Dahele M, Lock M, Yaremko B, Olson R, Liu M, Panarotto J, Griffioen GH, Gaede S, Slotman B and Senan S: Stereotactic ablative radiotherapy for comprehensive treatment of oligometastatic tumors (SABRCOMET): study protocol for a randomized phase II trial. BMC Cancer 12: 305, 2012.

18 Barnholtz-Sloan JS, Sloan AE, Davis FG, Vigneau FD, Lai P and Sawaya RE: Incidence proportions of brain metastases in patients diagnosed (1973 to 2001) in the Metropolitan Detroit Cancer Surveillance System. J Clin Oncol 22: 2865-2872, 2004.

19 Heon S, Yeap BY, Lindeman NI, Joshi VA, Butaney M, Britt GJ, Costa DB, Rabin MS, Jackman DM and Johnson BE: The impact of initial gefitinib or erlotinib versus chemotherapy on central nervous system progression in advanced non-small cell lung cancer with EGFR mutations. Clin Cancer Res 18: 44064414, 2012.

20 Park SJ, Kim HT, Lee DH, Kim KP, Kim SW, Suh C and Lee JS: Efficacy of epidermal growth factor receptor tyrosine kinase inhibitors for brain metastasis in non-small cell lung cancer patients harboring either exon 19 or 21 mutation. Lung Cancer 77: 556-560, 2012.
21 Wu YL, Zhou C, Cheng Y, Lu S, Chen GY, Huang C, Huang YS, Yan HH, Ren S, Liu Y and Yang JJ: Erlotinib as second-line treatment in patients with advanced non-small-cell lung cancer and asymptomatic brain metastases: a phase II study (CTONG0803). Ann Oncol 24: 993-999, 2013.

22 Kawaguchi Y, Hanaoka J, Hayashi H, Mizusaki N, Iihara H, Itoh $\mathrm{Y}$ and Sugiyama T: Clinical efficacy of afatinib treatment for a patient with leptomeningeal carcinomatosis. Chemotherapy 62 : 147-150, 2017.

23 Gerber NK, Yamada Y, Rimner A, Shi W, Riely GJ, Beal K, Yu HA, Chan TA, Zhang $\mathrm{Z}$ and $\mathrm{Wu}$ AJ: Erlotinib versus radiation therapy for brain metastases in patients with EGFR-mutant lung adenocarcinoma. Int J Radiat Oncol Biol Phys 89: 322-329, 2014.

24 Zhu Q, Sun Y, Cui Y, Ye K, Yang C, Yang D, Ma J, Liu X, Yu J and $\mathrm{Ge} \mathrm{H}$ : Clinical outcome of tyrosine kinase inhibitors alone or combined with radiotherapy for brain metastases from epidermal growth factor receptor (EGFR) mutant non small cell lung cancer (NSCLC). Oncotarget 8: 13304-13311, 2017.

25 Magnuson WJ, Lester-Coll NH, Wu AJ, Yang TJ, Lockney NA, Gerber NK, Beal K, Amini A, Patil T, Kavanagh BD, Camidge DR, Braunstein SE, Boreta LC, Balasubramanian SK, Ahluwalia MS, Rana NG, Attia A, Gettinger SN, Contessa JN, Yu JB and Chiang VL: Management of brain metastases in tyrosine kinase inhibitor-naive epidermal growth factor receptor-mutant nonsmall-cell lung cancer: A retrospective multi-institutional analysis. J Clin Oncol 35: 1070-1077, 2017.

Received October 15, 2017

Revised November 8, 2017 Accepted November 13, 2017 